\title{
DESIGN OF A SMART PREFABRICATED SANITISING CHAMBER FOR COVID-19 USING COMPUTATIONAL FLUID DYNAMICS
}

\author{
Yousef ABU-ZIDAN (1) 1 , Kate NGUYEN (D2) Priyan MENDIS ${ }^{1^{*}}$, \\ Sujeeva SETUNGE ${ }^{2}$, Hojjat ADELI ${ }^{3}$ \\ ${ }^{1}$ Department of Infrastructure Engineering, The University of Melbourne, Parkville, 3010 Victoria, Australia \\ ${ }^{2}$ School of Engineering, RMIT University, Melbourne, 3001 Victoria, Australia \\ ${ }^{3}$ Department of Civil, Environmental, and Geodetic Engineering, The Ohio State University, \\ Columbus, 43210 Ohio, USA
}

Received 29 June 2020; accepted 23 December 2020

\begin{abstract}
The novel coronavirus (SARS-CoV-2) has spread at an unprecedented rate, resulting in a global pandemic (COVID-19) that has strained healthcare systems and claimed many lives. Front-line healthcare workers are among the most at risk of contracting and spreading the virus due to close contact with infected patients and settings of high viral loads. To provide these workers with an extra layer of protection, the authors propose a low-cost, prefabricated, and portable sanitising chamber that sprays individuals with sanitising fluid to disinfect clothing and external surfaces on their person. The study discusses computer-aided design of the chamber to improve uniformity of sanitiser deposition and reduce discomfort due to excessive moisture. Advanced computational fluid dynamics is used to simulate the dispersion and deposition of spray particle, and the resulting wetting pattern on the treated person is used to optimise the chamber design.
\end{abstract}

Keywords: COVID-19, sanitising chamber, disinfection chamber, computational fluid dynamics (CFD), numerical simulation, computer aided design (CAD), portable, prefabricated.

\section{Introduction}

In the space of a few months, the novel coronavirus, named severe acute respiratory syndrome coronavirus 2 (SARS-CoV-2), has caught the entire world by surprise, spreading at an unprecedented rate across international borders of 187 countries and leading to a worldwide pandemic that has disrupted the global economy and claimed $1,430,822$ lives as of the 27th of November 2020 (Johns Hopkins University, 2020). This pandemic, named COVID-19 by the World Health Organisation (WHO), has exposed the unpreparedness of many countries in dealing with disease outbreaks (Jiang, 2020), highlighting the need to develop systems and tools to improve response to outbreaks of highly contagious diseases such as COVID-19 and others that may appear in the future. The two main strategies used to reduce transmission of COVID-19 have been through suppression and mitigation (Ferguson et al., 2020).

The highly contagious nature of SARS-CoV-2 is largely due to the mechanism through which it spreads from person to person via particles that are produced when an infected person speaks, coughs or sneezes (Hindson, 2020). These particles can land on nearby surfaces, where the virus can survive from a few hours to a few days depending on the environment and type of surface. During this time, SARS-CoV-2 is stable and may be contracted by touching the face after touching contaminated surfaces (van Doremalen et al., 2020). The virus may also land on clothing and exposed skin, where it can travel to new locations. This increases the risk of new infections and makes contact tracing more difficult. Frequent disinfection is therefore important to prevent spreading of the virus.

Among the most at risk of contracting and spreading the virus are frontline healthcare workers because of their close contact with infected persons and the settings of high viral loads in COVID-19 treatment facilities. Data released in Victoria, Australia showed that $10 \%$ of the state's confirmed cases are healthcare workers as of 1 April 2020 with clusters in the emergency department of hospitals across the state (Dow \& Sakkal, 2020). Additionally, the sudden onset of the virus has resulted in shortages of

*Corresponding author. E-mail: pamendis@unimelb.edu.au 
personal protective equipment (PPE) in many countries (both developed and developing), forcing frontline workers to reuse disposable PPEs such as gowns and masks. To provide an extra layer of protection for frontline workers, the authors propose a design for a prefabricated sanitising chamber that sprays individuals with a sanitising fluid to disinfect clothing and other external surfaces on their person. The low cost, prefabricated, and portable design of the chamber allows for quick manufacturing, transportation, and deployment in high-risk locations such as hospitals and virus testing centres. The chamber can also be used in places of large public gatherings (e.g. markets, schools, stadiums, etc.) as an additional measure to help reduce risk of recurring outbreaks as countries look to end lockdowns and resume economic activity.

\section{Design of the prefabricated sanitising chamber}

The proposed design of the prefabricated sanitising chamber (PSC) is shown in Figure 1. The chamber has a rectangular base of $0.9 \mathrm{~m}$ (width) by $1.2 \mathrm{~m}$ (length), with a height of $2.4 \mathrm{~m}$. Flexible polyvinyl chloride (PVC) lining encloses the sides and roof of the chamber, and PVC curtains are used in the entrance and exit. Misting nozzles are placed at the four corners of chamber and are horizontally levelled aligning with the diagonal lines of the chamber (Figure 1).

The chamber is operated by pumping sanitiser fluid through the misting nozzles to produce fine mists within the enclosed chamber. One person at a time enters the chamber from one side and stands under the sanitising mist. After spending a certain period of time in the chamber, and making a few rotations to ensure full coverage, the person exits the chamber from the other side.

The distribution of sanitising mist within the chamber largely dictates the efficacy of the chamber. A uniform distribution, both spatially and temporally, is highly desirable as this ensures effective sanitisation while reducing discomfort due to wetness of clothing. Hence, this study
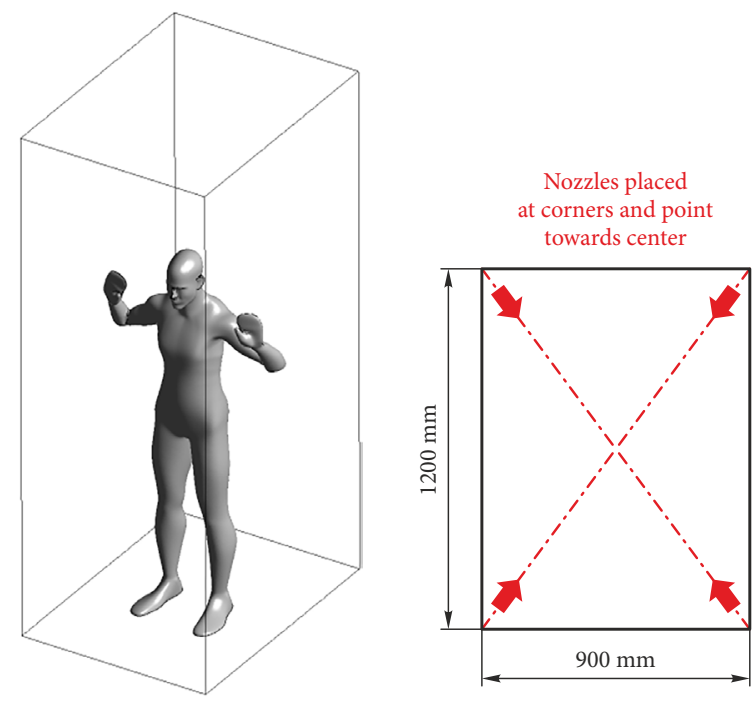

Figure 1. Isometric view (left) and plan view (right) of prefabricated sanitising chamber aims to optimise the design of the prefabricated sanitising chamber by improving the uniformity of sanitiser deposition on persons inside the chamber. Computational fluid dynamics (CFD) simulations are utilised for optimising the number of spray nozzles and their distribution within the PSC.

\section{Performance criteria}

To optimise the performance of the chamber, two limits of sanitiser concentration on the person are defined: a lower limit for viricidal efficacy, and an upper limit for avoiding discomfort due to excessive wetness. Sanitiser concentration on apparel is expressed in terms of grams per square meter (gsm). The performance of the chamber is optimised by maximising the percentage of the person's surface area with sanitiser concentration between the two limits.

The lower limit for viricidal efficacy is defined at $9 \%$ moisture content (i.e. "wetness") of the fabric by weight. This was selected based on in vitro experiments of hypochlorous acid delivered with a spray mechanism (Hakim et al., 2016). Hypochlorous acid is considered for use as a sanitising solution in the chamber due to its high viricidal efficacy against the COVID-19 virus, especially when applied by spray or fogger, and due to its availability, low-cost, and non-toxicity (Block \& Rowan, 2020).

The upper limit for wetness is defined based on the tolerable level of discomfort due to wetting of attire. To determine the condition of the fabric at different moisture contents, a mediumweight (180 gsm) medical garment of polyester (65\%) and cotton (35\%) blend (Figure 2) was wetted incrementally using a misting spray. For each case, the total weight of the garment was measured, and wetness was quantified as the percentage of absorbed liquid to the mass of the dry fabric. The condition of the garment at each wetness level is listed in Table 1.

Based on the condition of the garment in the trial, an upper limit of wetness was defined where it was estimated that a large portion of the population will experience significant discomfort. It should be noted that the criteria for wetness discomfort is highly subjective and varies depending on many parameters including environmental and personal conditions. However, for the purposes

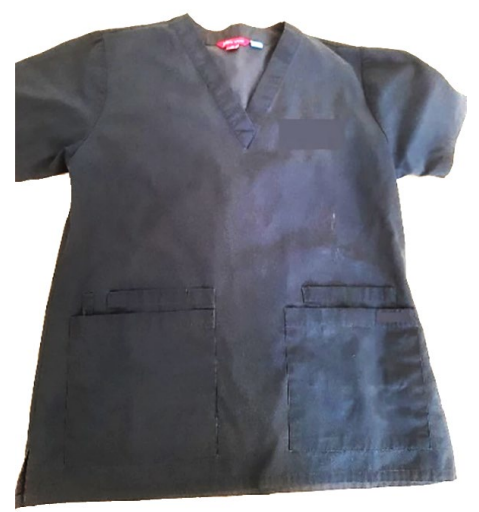

Figure 2. Mediumweight medical garment (180 gsm) 
Table 1. Condition of medical garment at various moisture contents

\begin{tabular}{|l|c|c|}
\hline \multicolumn{1}{|c|}{ Condition } & Total weight $(\mathrm{g})$ & Wetness \\
\hline dry & 225 & $0 \%$ \\
\hline slightly damp & 242 & $8 \%$ \\
\hline damp & 258 & $15 \%$ \\
\hline very damp & 270 & $20 \%$ \\
\hline wet & 320 & $42 \%$ \\
\hline very wet & 364 & $62 \%$ \\
\hline fully saturated & 499 & $122 \%$ \\
\hline
\end{tabular}

of the current investigation, the maximum tolerable level of wetness was drawn at the "very damp" category which corresponds to a wetness level of $20 \%$.

Additionally, an absorption ratio is defined to account for losses due to rebounding and evaporation of particles as these effects are not considered in the simulation. The absorption ratio was measured by placing the garment at a distance of $0.3 \mathrm{~m}$ from the misting nozzle. This is the approximate distance from the nozzles at the corners of the chamber, to the person standing in the middle. The garment was positioned to fully capture the flow from the nozzle and the increase in the mass of the garment was measured over a given period. The absorption ratio is calculated as the ratio of liquid absorption rate by the garment to the total flow rate from the nozzle. This value was calculated to be 0.64 .

Given an upper limit of wetness of $20 \%$, and the absorption rate of 0.64 , the maximum sanitiser concentration for the mediumweight garment is $180 \mathrm{gsm} \times 0.2 / 0.64=$ 56. Similar computations can be performed for the lower limit of $9 \%$ and for various types of garments. Table 2 lists thresholds of sanitiser concentration for clothing configurations considered in this study.

\section{Flow properties of misting nozzle}

For the design of the misting system, a generic plain-orifice atomiser nozzle is considered due to their wide availability and low cost (Figure 3). For this type of nozzle, the liquid is ejected at a high velocity through a small round orifice. These nozzles are available with various internal orifice diameters typically ranging from $0.2-1 \mathrm{~mm}$. Small nozzles produce a finer mist at a lower pressure and flow rate compared to larger nozzles, but they are more susceptible to blockage from fine particles in the flow. Generally,
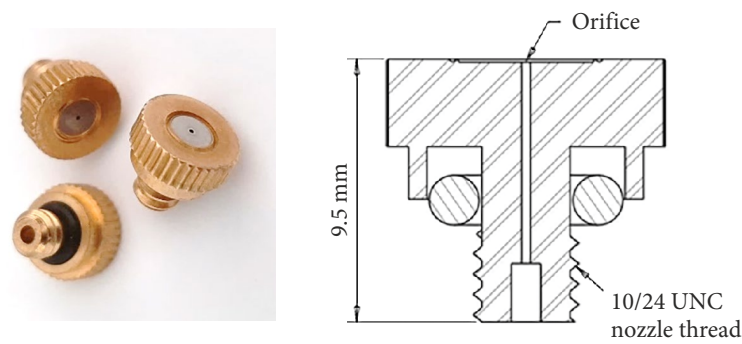

Figure 3. Plain-orifice misting nozzle used in the model (Mistcooling Inc., 2020)

a minimum diameter of $0.3 \mathrm{~mm}$ is recommended to avoid blockage (Lefebvre \& McDonell, 2017).

The flow performance of these nozzles is primarily influenced by the flow pressure. Larger diameter nozzle requires larger pressures (and higher flow rates) to achieve a fine mist, which requires a more powerful pump.

\subsection{Experimental investigation of nozzle flow}

To validate the flow properties of the nozzle in the simulation, an experimental investigation is performed for an orifice diameter of $0.3 \mathrm{~mm}$, and the flow rate was varied at 2,3 , and 4.4. L/hr. The resulting flow trajectories and spray angles are shown in Figure 4 and Figure 5.

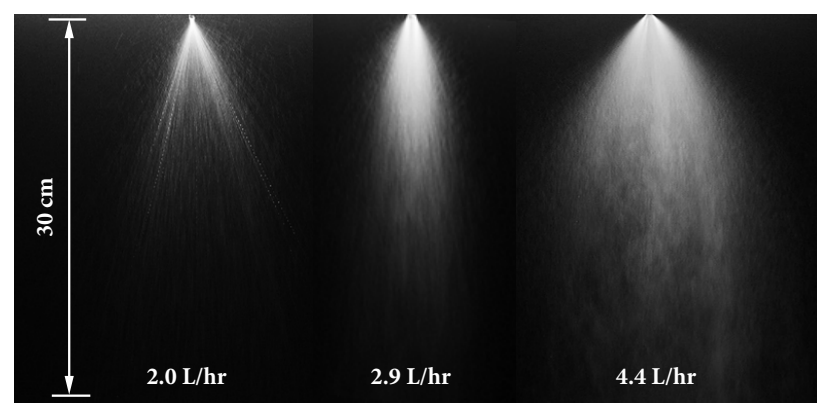

Figure 4. Nozzle flow trajectory for various flow rates

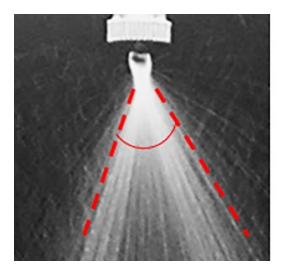

$2.0 \mathrm{~L} / \mathrm{hr}$ $\alpha=50^{\circ}$

Figure 5. Nozzle spray angle for various flow rates

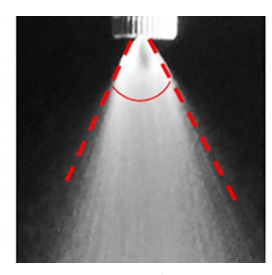

$2.9 \mathrm{~L} / \mathrm{hr}$
$\alpha=55^{\circ}$

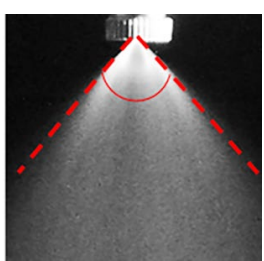

$4.4 \mathrm{~L} / \mathrm{hr}$
$\alpha=90^{\circ}$

Table 2. Upper and lower wetness limits for various outfits

\begin{tabular}{|l|c|c|c|}
\hline \multicolumn{1}{|c|}{ Outfit } & $\begin{array}{c}\text { Fabric density } \\
\text { (gsm) }\end{array}$ & $\begin{array}{c}\text { Lower limit for } \\
\text { viricidal efficacy } \\
\text { (gsm) }\end{array}$ & $\begin{array}{c}\text { Upper limit } \\
\text { for avoiding } \\
\text { discomfort (gsm) }\end{array}$ \\
\hline Lightweight garment & $140 \mathrm{gsm}$ & 20 & 44 \\
\hline Mediumweight garment & $180 \mathrm{gsm}$ & 25 & 56 \\
\hline $\begin{array}{l}\text { Mediumweight scrubs with lightweight garment worn underneath } \\
\text { or } \\
\text { Heavyweight (scrubs) }\end{array}$ & $320 \mathrm{gsm}$ & 45 & 100 \\
\hline
\end{tabular}


It can be seen in Figure 4 and Figure 5 that at a flow rate of $2.0 \mathrm{~L} / \mathrm{hr}$, the flow pressure was not sufficient to produce a fine mist. Instead, the nozzle produced a weak spray characterised by larger droplets and a smaller spray angle 50 degrees. The middle case with a flow rate of $2.9 \mathrm{~L} / \mathrm{hr}$ produced a similar flow with a slightly finer mist and a spray angle of 55 degrees. However, at $4.4 \mathrm{~L} / \mathrm{hr}$, the nozzle demonstrated a significantly different behaviour, producing a fine mist with a wide spray angle of 90 degrees. This flow is desirable for improving uniformity of flow in the chamber. The flow rate of $4.4 \mathrm{~L} / \mathrm{hr}$ was achieved at a pressure of $470 \mathrm{kPa}$, which was the maximum pressure possible with the equipment used.

Validation of the numerical model is performed by comparing the wetting pattern produced by the nozzle at various heights. In the experiment, wetting patterns were obtained by placing a piece of fabric at specific distances from the nozzle. A digital camera is used to record the wetting patterns and resulting images are processed to correct perspective distortion and to highlight wetting areas. The resulting wetting patterns are shown in Figure 6. The wetting area was estimated for each case, and the equivalent circular radius is calculated. These results are were used to validate the numerical model of the nozzle.

\subsection{Validation of numerical model of nozzle}

Before numerical simulations of the chamber were performed, the nozzle properties were validated with experimental results. Simulations were performed in an empty
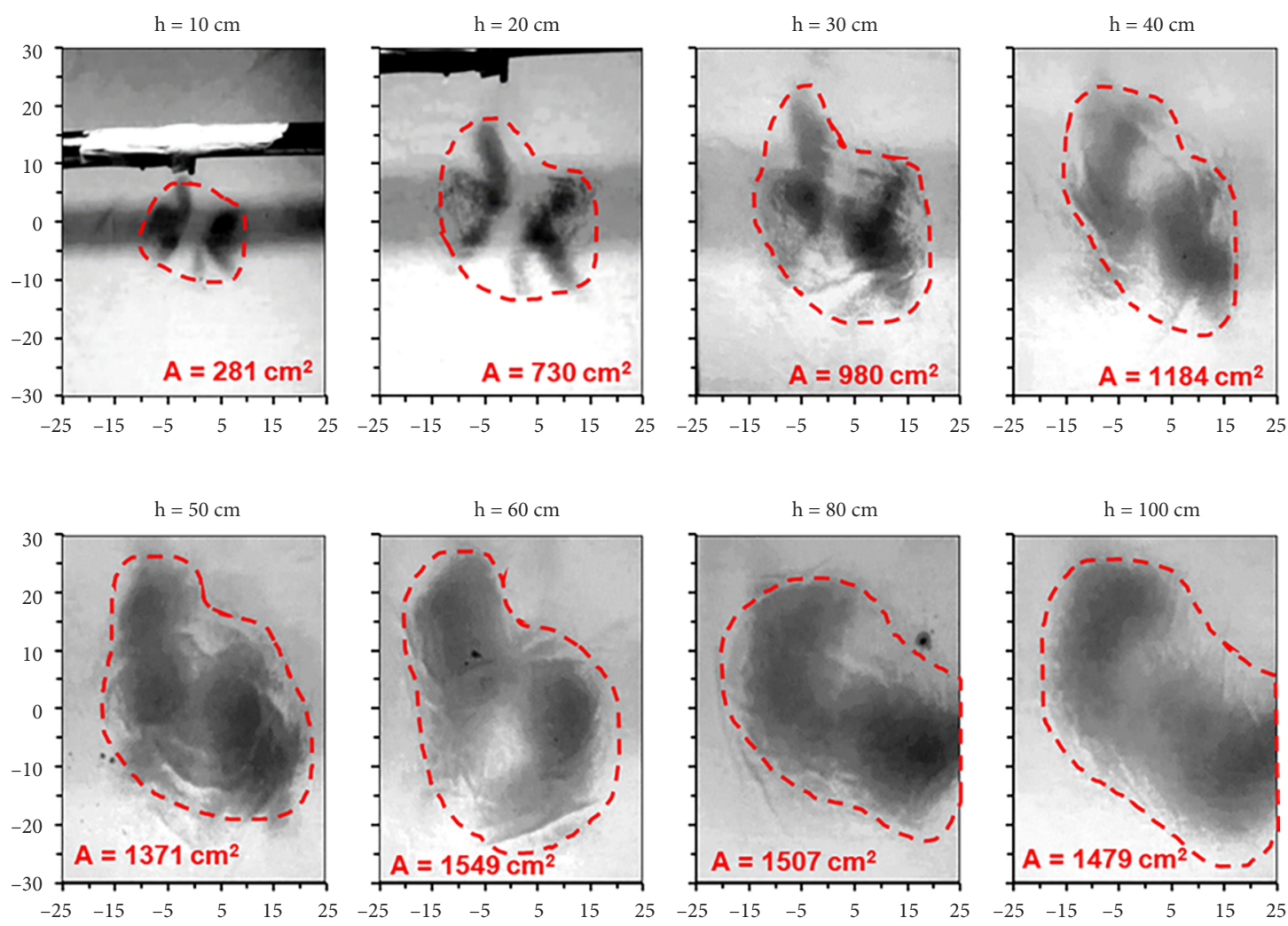

computational domain with a single particle injector that simulates the nozzle. Properties of the injected particles were predicted using the plain-orifice atomiser model provided in FLUENT that was developed based on empirical studies (Lichtarowicz et al., 1965; Nurick, 1976; Ranz, 1958).

The plain-orifice model uses input parameters of nozzle geometry and flow rate to compute the exit velocity, spray angle, and particle size distribution. The flow rate and nozzle diameter were set at $4.4 \mathrm{~L} / \mathrm{hr}$ and $0.3 \mathrm{~mm}$ respectively. A correlation constant for the spray angle (Ranz, 1958) was calibrated to achieve a spray angle of $90^{\circ}$, which corresponds to the validation experiment. The plain-orifice model resulted in a Rosin-Rammler droplet size distribution with minimum, mean, and maximum diameters of $3 \mu \mathrm{m}, 107 \mu \mathrm{m}$, and $300 \mu \mathrm{m}$, respectively. These values fall within the expected size range reported in experimental investigation of high-pressure water-mists generated using solid-cone plain-orifice nozzles (Santangelo, 2010). The number of discrete particle streams for the nozzle was set to 500. The spherical drag law was used to model the interaction of the discrete particles with the airflow, and the effect of turbulence on particle trajectory is estimated with stochastic tracking based on random walk theory (ANSYS Inc., 2013).

Wetting patterns from the numerical model were extracted at equivalent heights to the experiment and the results are compared in Figure 7, which shows good agreement with an average difference of $7 \%$ between numerical and experimental values.

Figure 6. Wetting patterns at distance (h) from the nozzle 


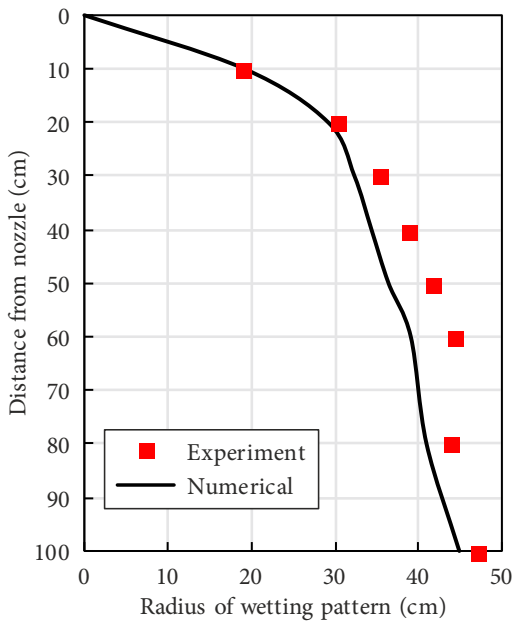

Figure 7. Validation of nozzle flow from numerical model

\section{Details of numerical model}

Simulations of the chamber were performed using CFD software FLUENT with a steady-state RANS model for the continuous phase to simulate airflow and LagrangianEulerian method for tracking movement of discrete particles. Additionally, the Eulerian Wall Film interface model was used to simulate deposition of spray particles on the person.

\subsection{Computational grid and boundary conditions}

A rectangular computational domain was constructed with dimensions equivalent to the chamber $(0.9 \times 1.2 \times 2.4 \mathrm{~m})$, and a geometric model of a $1.75 \mathrm{~m}$ tall person was placed in the middle of the domain. An unstructured Cartesian mesh was generated with $0.5 \times 10^{6}$ elements, and 5 inflation layers of prismatic cells were used to capture the boundary layer at the walls of the domain and on the person (Figure 8). For the continuous phase, a no-slip wall boundary condition was specified at the domain boundaries and on the person.

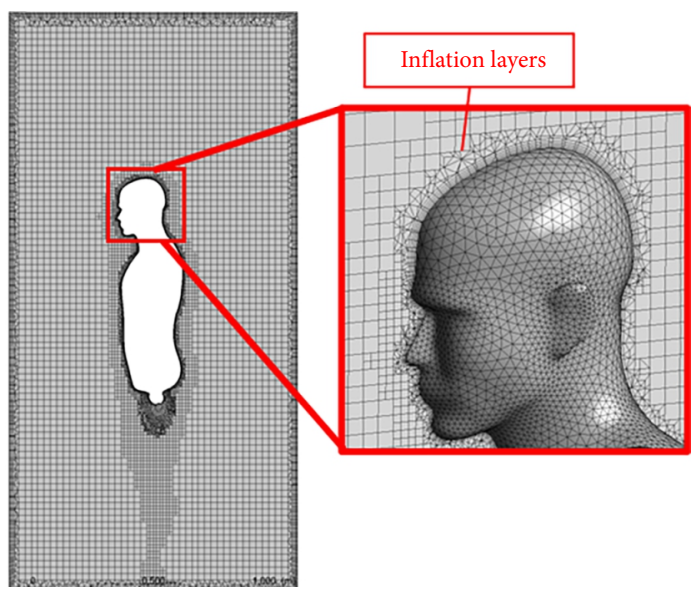

Figure 8. Computational mesh on a vertical plane and person. Total cell count is $0.5 \times 10^{6}$

\subsection{Solver settings}

Turbulence in the continuous phase was modelled using the realisable k- $\varepsilon$ model (Shih et al., 1995), and the standard wall function (Launder \& Spalding, 1974) was used to model boundary layers adjacent to the chamber walls and on the person. The continuous phase was solved using the SIMPLE pressure-based segregated solver (ANSYS Inc., 2013), and second order discretisation schemes were used for all flow variables. Tracking of discrete particles was performed for every 10 iterations of the continuous phase, while the wall film model was updated for each iteration of the continuous phase. The timestep of the wall film model was set to $0.1 \mathrm{~s}$ per iteration. To reduce complexity of the wall film model, secondary effects due to particle rebound, splashing, and evaporation were ignored in the simulations. These effects were accounted for in the postprocessing stage using an absorption ratio as discussed later. Simulations are run initially for 500 iterations with the wall-film model disabled in order to initialize the continuous and discrete phases. Then, the wall film is enabled, and the solution is run for a further 300 iterations, resulting in a total wall film time of $30 \mathrm{~s}$. Simulations were performed on a single computing node with two 6-core Intel(R) Xeon(R) E5-2620 v3 processors @ 2.40 GHz and a total RAM of $64 \mathrm{~GB}$. The average computational time for each case was $902.4 \mathrm{~s}$.

\section{Results and analysis}

Design cases for the nozzle layouts were generated by discretising the height of the chamber into $0.3 \mathrm{~m}$ increments. All possible combination nozzle layouts were considered provided that adjacent nozzle layers were at least $0.6 \mathrm{~m}$ apart. This resulted in a total of 33 possible combinations. The time in the chamber was varied at 5 second increment for a total time of 30 seconds, and three types of clothing were considered as listed in Table 2. Combination of all parameter resulted in a total of 594 data points.

The resulting wetting patterns for a sample of cases with 1,2, and 3 nozzle layers are presented in Figure 9. Blue zones indicate regions of high concentration of sanitiser deposition while white zones indicate areas of low concentration. Figure 9 demonstrates that increasing the number of nozzle layers in the chamber generally tends to improve the uniformity of sanitiser distribution. The case with three layers of misting nozzles achieved a more uniform sanitiser distribution, compared to the case with 1 and 2 nozzle layers especially in the main body and legs. White areas on arms that showed a lower concentration of sanitiser may inhibit viral efficacy. To improve sanitiser distribution, it is recommended that the person perform a full rotation inside the PSC during the misting cycle.

For all data point in the study, the performance of the chamber was quantified by calculating the proportion of surface area on the person with sanitiser distribution to the wetness limits defined in Table 2. Figure 10 presents 
the results for the case of medium density clothing. The horizontal axis plots the percentage of the person's surface area with sanitiser concentration above the minimum limit for viricidal efficacy. This measures the performance of the chamber for effective sanitisation without consideration of discomfort. From Figure 10 it can be seen with increasing time in the chamber, all data points travel to the right indicating a larger portion of the person is exceeding the threshold for effective sanitising. This levels off at around $70 \%$ of the person experiencing effective sanitisation.

The vertical axis in Figure 10 plots the percentage of the person's surface area that is sanitised but does exceed the threshold for discomfort. This quantifies the combined performance of effective sanitisation and comfort. In can be seen that the data points initially travel upwards, but eventually reach a peak and move downwards as upper limits for comfort are exceeded on a larger portion of the person.

The optimal nozzle configuration of the chamber is selected by sorting the data based on performance criteria,
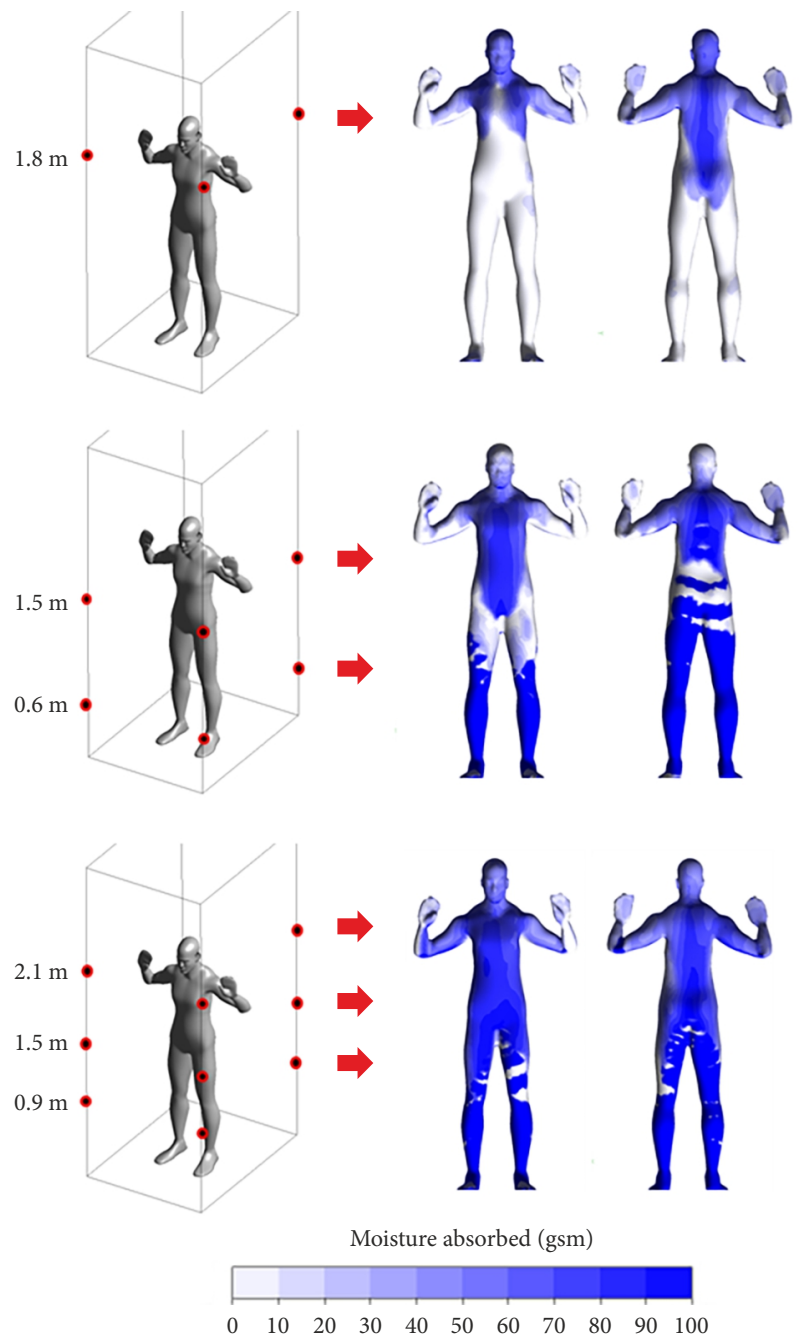

Figure 9. Wetting pattern on targeted person's garment for different nozzle layouts. Results correspond to 30 second exposure to sanitising mist which revealed two optimal configurations. A case with three layers of nozzles at heights of $0.9,1.5$, and $2.1 \mathrm{~m}$, and a case with four layers at heights of $0.3,0.9,1.5,2.1 \mathrm{~m}$. Both cases showed comparable performance, so the case with three layers was selected due to its lower sanitiser consumption. The wetting pattern for this case is presented in Figure 9 (bottom).

The performance of the optimised case at various times and clothing conditions is plotted in Figure 11. The figure shows that for light and medium weight clothing, the chamber was generally effective in exceeding limits for viricidal efficacy at $>70 \%$ for cases with the medium and lightweight clothing, and around $65 \%$ for the case of heavy clothing. The lower value for the heavy clothing is due to the limits for viricidal efficacy being defined in terms of moisture contents of fabric density (Table 2). This results in a higher sanitiser concentration required for heavier garments, which is a conservative approximation considering that sanitisation is typically required on the outer portion of heavy garments.

Achieving combined performance of sanitisation and comfort (i.e. sanitised but not too wet) was challenging particularly for light and medium clothing. The magnitude of peak combined performance was quite low with values of $19 \%, 29 \%$, and $51 \%$ for light, medium and heavy clothing respectively, compared with a 100\% target for perfectly uniform sanitiser distribution in the chamber. The high-pressure flow required for generating the mist, combined with the close distance between the person and the nozzles, make it very difficult to achieve perfectly uniform distributions of spray deposition within the compact space of the chamber. Varying the flow rate of individual nozzles or nozzle layers could improve performance, but these can be challenging to implement in practice. Alternatively, performance can be improved by considering more expensive spraying systems such as air atomising nozzles that can achieve a finer mist, but these would add to the complexity and cost of the chamber.

\section{Limitations and future work}

Several limitations of the study should be noted. The simulation of the continuous phase was performed in steadystate RANS that does not accurately capture the highly turbulent flow within the chamber. The chamber was assumed to be sealed with no interfering airflow from the external environment, and the discrete model does not account for complex effects such as breakup and collision of droplets. In the wall film model, the effect of evaporation and splashing were ignored, as well as tracking of particles on the surface due to gravity and diffusion through the fabric. These simplifying assumptions were necessary to reduce the complexity and computational time of the model.

Moreover, validation was performed using simple imaging techniques which have limitations. Most notably, the experiment did not allow quantification of spray concentrations and only provided approximate values of wetted 

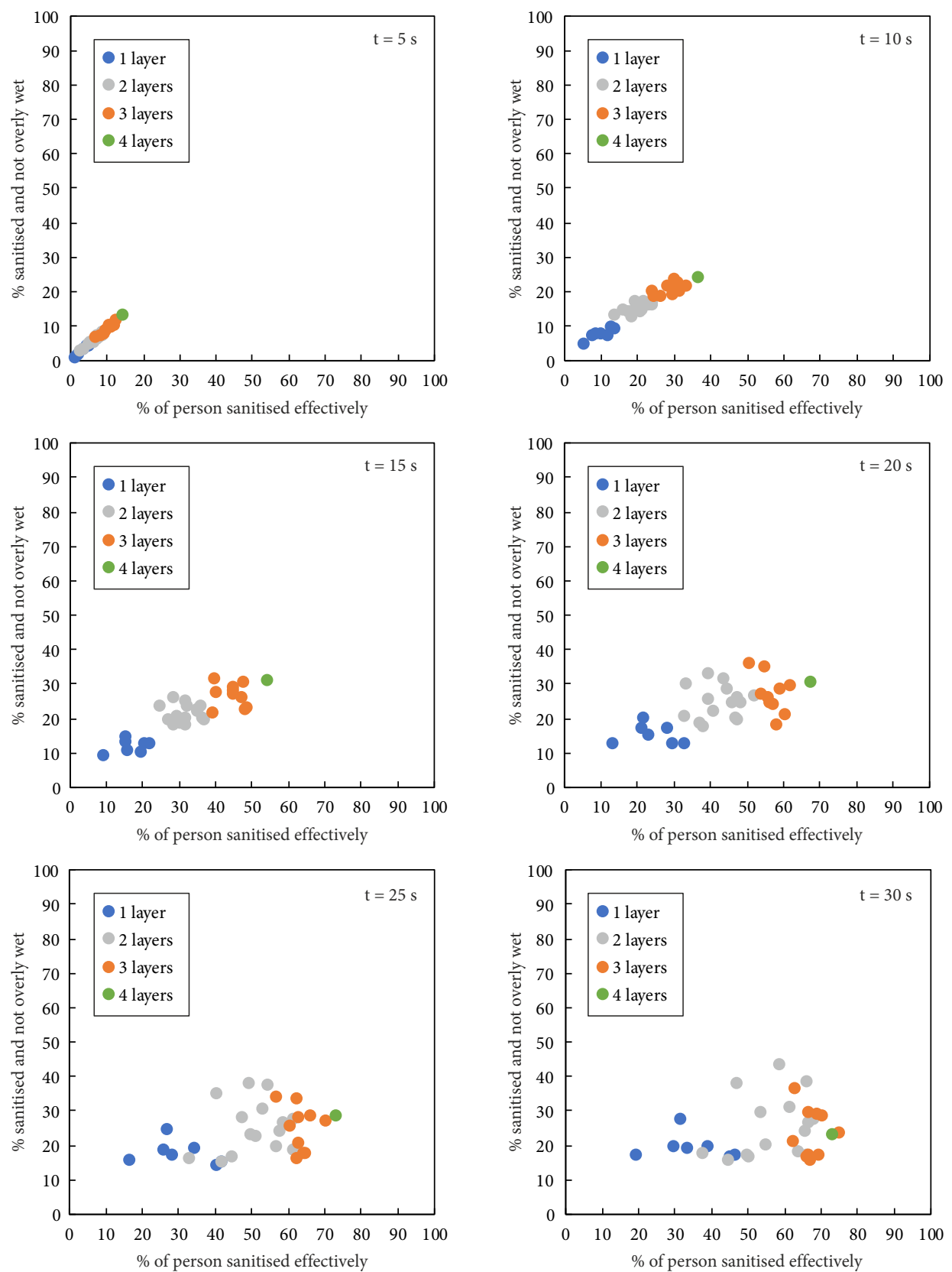

Figure 10. Performance of chamber for various nozzle layouts. Results shown are for mediumweight clothing
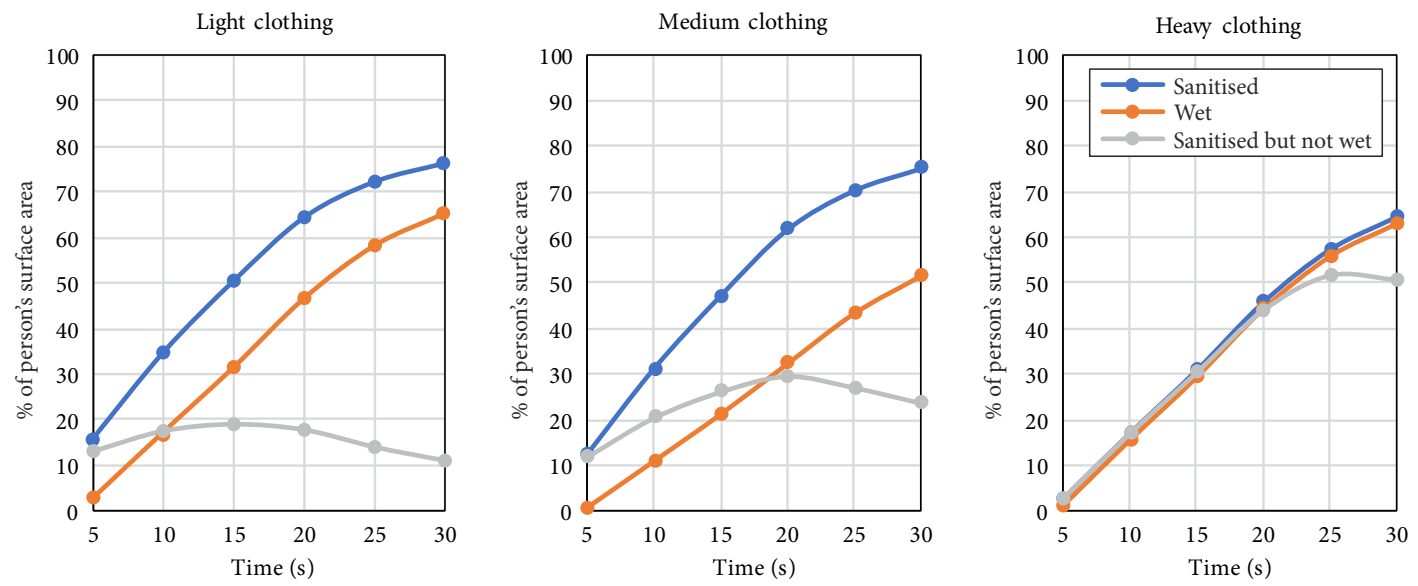

Figure 11. Performance of optimised chamber for different clothing 
areas. Advanced methods are available for rigorously validating nozzle properties such as direct image correlation (Take, 2015) and particle image velocimetry (Zhu et al., 2017) but these require specialised tools not available to the authors at the time of performing the study. Nonetheless, for the purposes of guiding the preliminary design of the PSC, the low-cost experimental approach used in this study was useful for validating general flow behaviour of the nozzle. Field tests will be performed to validate the performance of the actual chamber prior to deployment in parallel with efficacy testing of SARS-CoV-2.

Future work will focus on utilising machine learning to further improve the design of the SPSC. The current study has demonstrated the feasibility of producing large datasets for this application. This is due to the relatively low cost of the computational model and the large number of data points generated in the postprocessing stages. Machine learning algorithms have been developed for design optimisation of prefabricated and portable structures (Chikahiro et al., 2019; Zawidzki \& Jankowski, 2019) as well as for monitoring the health of structures (Rafiei \& Adeli, 2017, 2018b). Such methods can help optimise the structural design of the SPCS. For the design of the spray system, the current study only considered a limited number of design parameters relating to the nozzle configuration. Other parameters not considered in the current study, such as nozzle orientation could be optimised in future studies with the aid of machine learning algorithms. Moreover, machine learning methods, such as genetic algorithms, may help improve convergence of CFD simulations and reduce computational cost by optimising numerical parameters of the CFD solver (Ghorbel et al., 2019). Lowering the computational cost of CFD would allow for an even larger number of simulations cases to be performed.

Machine learning could also be used with experimental datasets to generate models for predicting the performance of the spraying system. This approach was demonstrated by Junior et al. (2017), who utilised artificial neural networks and regression trees to generate models for predicting the concentration and distribution of pesticide spray for protecting crops. A similar study by Guo et al. (2020) also used machine learning algorithms and experimental datasets to quantitatively predict the droplet size and deposition distribution. These models were used to optimise the spacing between the nozzles and distance from the target to improve the uniformity of particle depositions.

In addition to improving the design of the prefabricated structure and spraying system, machine learning is proposed to optimise production and delivery of the SPCS. Machine learning algorithms have been developed for improving cost estimation in engineering projects (Rafiei \& Adeli, 2018a; Shahrara et al., 2017), for accelerating project schedules (Wang et al., 2016a, 2016b) and for reducing the level of finished inventory of prefabricated components (Ko, 2010). Such approaches would help facilitate efficient upscaling of manufacturing and delivery of the SPCS while reducing cost and time of production.

\section{Conclusions}

To provide an extra layer of protection against the spread of COVID-19 and similar viruses, the authors propose a smart, prefabricated, and portable sanitising chamber that can be easily manufactured and deployed in hospitals and other places of public gatherings. The study aimed to optimise the performance of the prefabricated sanitising chamber (PSC) by improving the uniformity of sanitiser distribution on the persons using the chamber, which leads to improved effectiveness of sanitization while reducing consumption of sanitiser fluid.

A generic plain-orifice misting nozzle was selected for the study due to its low cost and wide availability. The properties of the misting nozzles were measured experimentally and used to validate the numerical model. Results showed good agreement with an average difference of $7 \%$ between experimental and numerical values. The nozzle achieved a fine mist at a flow rate of $4.4 \mathrm{~L} / \mathrm{hr}$ with a spray angle of 90 degrees.

The numerical model was then used to optimise the nozzle layout by varying the number of nozzles and their position within the chamber. Parameters considered include nozzle layout, type of clothing, and time spend in chamber. Results indicate that uniformity of sanitiser distribution could be improved by increasing the number of nozzle layers in the chamber. The optimal layout configuration involved three nozzle layers located at heights of 0.9 $\mathrm{m}, 1.5 \mathrm{~m}$, and $2.1 \mathrm{~m}$.

The performance of the chamber was quantified by calculating the proportion of surface area on the person with sanitiser concentration above the minimum limit required for effective sanitisation. The optimised case achieved good sanitisation performance with around $70 \%$ of the person experiencing sanitiser concentration above the defined threshold for viricidal efficacy Nonetheless, achieving combined performance of sanitisation and comfort was challenging with the low-cost misting nozzle considered in this study, particularly for lightweight clothing.

To further enhance the performance of the chamber, future studies are recommended to investigate the effect of other factors such as nozzle flow rate and orientation, and forced ventilation inside the chamber. The authors are currently working with medical experts to establish safe levels of exposure to sanitising mist, as well as assessing the microbicidal efficacy of the sanitising mist against pathogens including SARS-CoV-2 virus and highly resistant bacteria.

\section{Acknowledgements}

The manuscript has been prepared from the engineering package of the multidisciplinary project between RMIT University (School of Engineering) and the University of Melbourne (ARC Centre for Advanced Manufacturing of Prefabricated Housing, Peter Doherty Institute for Infection and Immunity and Centre of Epidemiology and Biostatistics). 


\section{Funding}

The work was supported by ARC DECRA Grant [ID: DE190100217], RMIT University, and the ARC Training Centre for Advanced Manufacturing of Prefabricated Housing [Grant ID: IC150100023] of the Department of Infrastructure Engineering, the University of Melbourne.

\section{Author contributions}

YA designed and performed the study and drafted the manuscript. KN and PM conceived the study and edited the manuscript. SS and HA revised the manuscript.

\section{Disclosure statement}

The authors have no conflict of interest to declare.

\section{References}

ANSYS Inc. (2013). ANSYS Fluent theory guide. USA.

Block, M. S., \& Rowan, B. G. (2020). Hypochlorous acid: A review. Journal of Oral and Maxillofacial Surgery, 78(9), 1461-1466. https://doi.org/10.1016/j.joms.2020.06.029

Chikahiro, Y., Ario, I., Pawlowski, P., Graczykowski, C., \& Holnicki-Szulc, J. (2019). Optimization of reinforcement layout of scissor-type bridge using differential evolution algorithm. Computer-Aided Civil and Infrastructure Engineering, 34(6), 523-538. https://doi.org/10.1111/mice.12432

Dow, A., \& Sakkal, P. (2020, April 1). Eighty Victorian healthcare workers test positive for coronavirus. The Age. https://wwwtheage-com-au.eu1.proxy.openathens.net/national/victoria/ eighty-victorian-healthcare-workers-test-positive-for-coronavirus-20200401-p54g55.html

Ferguson, N., Laydon, D., Nedjati Gilani, G., Imai, N., Ainslie, K., Baguelin, M., Bhatia, S., Boonyasiri, A., Cucunuba Perez, Z., Cuomo-Dannenburg, G., Dighe, A., Dorigatti, I., Fu, H., Gaythorpe, K., Green, W., Hamlet, A., Hinsley, W., Okell, L., Van Elsland, S., Thompson, H., Verity, R., Volz, E., Wang, H., Wang, Y., Walker, P., Walters, C., Winskill, P., Whittaker, C., Donnelly, C., Riley, S., \& Ghani, A. (2020). Report 9: Impact of non-pharmaceutical interventions (NPIs) to reduce COVID-19 mortality and healthcare demand.

https://doi.org/10.25561/77482

Ghorbel, H., Zannini, N., Cherif, S., Sauser, F., Grunenwald, D., Droz, W., Baradji, M., \& Lakehal, D. (2019). Smart adaptive run parameterization (SArP): enhancement of user manual selection of running parameters in fluid dynamic simulations using bio-inspired and machine-learning techniques. Soft Computing, 23(22), 12031-12047.

https://doi.org/10.1007/s00500-019-03761-6

Guo, H., Zhou, J., Liu, F., He, Y., Huang, H., \& Wang, H. (2020). Application of machine learning method to quantitatively evaluate the droplet size and deposition distribution of the UAV spray nozzle. Applied Sciences, 10(5), 1759. https://doi.org/10.3390/app10051759

Hakim, H., Alam, M. S., Sangsriratanakul, N., Nakajima, K., Kitazawa, M., Ota, M., Toyofuku, C., Yamada, M., Thammakarn, C., Shoham, D., \& Takehara, K. (2016). Inactivation of bacteria on surfaces by sprayed slightly acidic hypochlorous acid water: in vitro experiments. The Journal of Veterinary Medical Science, 78(7), 1123-1128.

https://doi.org/10.1292/jvms.16-0075
Hindson, J. (2020). COVID-19: faecal-oral transmission? Nature Reviews Gastroenterology \& Hepatology, 17(5), 259-259. https://doi.org/10.1038/s41575-020-0295-7

Jiang, S. (2020). Don't rush to deploy COVID-19 vaccines and drugs without sufficient safety guarantees. Nature, 579, 321321. https://doi.org/10.1038/d41586-020-00751-9

Johns Hopkins University. (2020). COVID-19 Dashboard by the Center for Systems Science and Engineering (CSSE) at Johns Hopkins University (JHU). https://coronavirus.jhu.edu/map. html

Junior, C. R., Gomes, P. H., Mano, L. Y., de Oliveira, R. B., de Carvalho, A. C. P. de L. F., \& Faiçal, B. S. (2017). A machine learning-based approach for prediction of plant protection product deposition [Conference presentation]. 2017 Brazilian Conference on Intelligent Systems (BRACIS), Uberlandia, Brazil. https://doi.org/10.1109/BRACIS.2017.26

Ko, C. H. (2010). An integrated framework for reducing precast fabrication inventory. Journal of Civil Engineering and Management, 16(3), 418-427. https://doi.org/10.3846/jcem.2010.48

Launder, B. E., \& Spalding, D. B. (1974). The numerical computation of turbulent flows. Computer Methods in Applied Mechanics and Engineering, 3(2), 269-289.

https://doi.org/10.1016/0045-7825(74)90029-2

Lefebvre, A. H., \& McDonell, V. G. (2017). Atomization and sprays. CRC Press. https://doi.org/10.1201/9781315120911

Lichtarowicz, A., Duggins, R. K., \& Markland, E. (1965). Discharge coefficients for incompressible non-cavitating flow through long orifices. Journal of Mechanical Engineering Science, 7(2), 210-219.

https://doi.org/10.1243/JMES_JOUR_1965_007_029_02

Mistcooling Inc. (2020). Dimensions of misting nozzle. https:// www.mistcooling.com/mist-nozzles-10-24-thread.html

Nurick, W. H. (1976). Orifice cavitation and its effect on spray mixing. Journal of Fluids Engineering, 98(4), 681-687. https://doi.org/10.1115/1.3448452

Rafiei, M. H., \& Adeli, H. (2017). A novel machine learningbased algorithm to detect damage in high-rise building structures. The Structural Design of Tall and Special Buildings, 26(18), e1400. https://doi.org/10.1002/tal.1400

Rafiei, M. H., \& Adeli, H. (2018a). Novel machine-learning model for estimating construction costs considering economic variables and indexes. Journal of Construction Engineering and Management, 144(12), 04018106.

https://doi.org/10.1061/(ASCE)CO.1943-7862.0001570

Rafiei, M. H., \& Adeli, H. (2018b). A novel unsupervised deep learning model for global and local health condition assessment of structures. Engineering Structures, 156, 598-607. https://doi.org/10.1016/j.engstruct.2017.10.070

Ranz, W. E. (1958). Some experiments on orifice sprays. The Canadian Journal of Chemical Engineering, 36(4), 175-181. https://doi.org/10.1002/cjce.5450360405

Santangelo, P. E. (2010). Characterization of high-pressure watermist sprays: Experimental analysis of droplet size and dispersion. Experimental Thermal and Fluid Science, 34(8), 13531366. https://doi.org/10.1016/j.expthermflusci.2010.06.008

Shahrara, N., Çelik, T., \& Gandomi, A. H. (2017). Gene expression programming approach to cost estimation formulation for utility projects. Journal of Civil Engineering and Management, 23(1), 85-95. https://doi.org/10.3846/13923730.2016.1210214

Shih, T.-H., Liou, W. W., Shabbir, A., Yang, Z., \& Zhu, J. (1995). A new k- $\epsilon$ eddy viscosity model for high reynolds number turbulent flows. Computers \& Fluids, 24(3), 227-238. https://doi.org/10.1016/0045-7930(94)00032-T 
Take, W. A. (2015). Thirty-Sixth Canadian Geotechnical Colloquium: Advances in visualization of geotechnical processes through digital image correlation. Canadian Geotechnical Journal, 52(9), 1199-1220.

https://doi.org/10.1139/cgj-2014-0080

van Doremalen, N., Bushmaker, T., Morris, D. H., Holbrook, M. G., Gamble, A., Williamson, B. N., Tamin, A., Harcourt, J. L., Thornburg, N. J., Gerber, S. I., Lloyd-Smith, J. O., de Wit, E., \& Munster, V. J. (2020). Aerosol and surface stability of SARSCoV-2 as compared with SARS-CoV-1. New England Journal of Medicine, 382(16), 1564-1567.

https://doi.org/10.1056/NEJMc2004973

Wang, C., Abdul-Rahman, H., \& Ch'ng, W. S. (2016a). Ant colony optimization (ACO) in scheduling overlapping architectural design activities. Journal of Civil Engineering and Management, 22(6), 780-791.

https://doi.org/10.3846/13923730.2014.914100

Wang, C., Abdul-Rahman, H., \& Chow, P. S. (2016b). Development and test run of civil engineering schedule acceleration model through ant colony optimization. Journal of Civil Engineering and Management, 22(8), 1009-1020. https://doi.org/10.3846/13923730.2014.945954

Zawidzki, M., \& Jankowski, Ł. (2019). Multiobjective optimization of modular structures: Weight versus geometric versatility in a Truss-Z system. Computer-Aided Civil and Infrastructure Engineering, 34(11), 1026-1040. https://doi.org/10.1111/mice.12478

Zhu, P., Wang, X. S., Li, G. C., Liu, Y. P., Kong, X. X., Huang, Y. Q., Zhao, X. D., \& Yuan, J. W. (2017). Experimental study on interaction of water mist spray with high-velocity gas jet. Fire Safety Journal, 93, 60-73.

https://doi.org/10.1016/j.firesaf.2017.08.005 